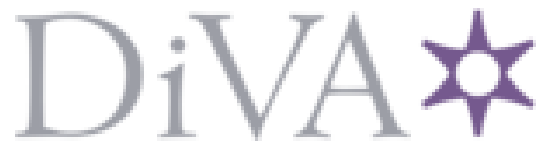

http://www.diva-portal.org

Preprint

This is the submitted version of a chapter published in Studies in Computational Intelligence: Computational Intelligence. IJCCI 2019.

Citation for the original published chapter:

Palm, R., Lilienthal, A J. (2021)

Fuzzy Geometric Approach to Collision Estimation Under Gaussian Noise in Human-

Robot Interaction

In: Merelo J.J., Garibaldi J., Linares-Barranco A., Warwick K., Madani K. (ed.), Studies in Computational Intelligence: Computational Intelligence. IJCCI 2019. Cham:

Springer

Studies in Computational Intelligence

https://doi.org/10.1007/978-3-030-70594-7_8

N.B. When citing this work, cite the original published chapter.

Permanent link to this version:

http://urn.kb.se/resolve?urn=urn:nbn:se:oru:diva-97012 


\title{
Fuzzy Geometric Approach to Collision Estimation Under Gaussian Noise in Human-Robot Interaction
}

\author{
Rainer Palm ${ }^{1}$ and Achim J. Lilienthal ${ }^{2}$ \\ 1 AASS, Dept. of Technology Orebro University SE-70182 Orebro, Sweden \\ rub.palm@t-online.de \\ 2 AASS, Dept. of Technology Orebro Universit, SE-70182 Orebro, Sweden \\ achim.lilienthal@oru.se
}

\begin{abstract}
Humans and mobile robots while sharing the same work areas require a high level of safety especially at possible intersections of trajectories. An issue of the human-robot navigation is the computation of the intersection point in the presence of noisy measurements or fuzzy information. For Gaussian distributions of positions/orientations (inputs) of robot and human agent and their parameters the corresponding parameters at the intersections (outputs) are computed by analytical and fuzzy methods. This is done both for the static and the dynamic case using Kalman filters for robot/human positions and orientations and thus for the estimation of the intersection positions. For the overdetermined case (6 inputs, 2 outputs) a so-called 'energetic' approach is used for the estimation of the point of intersection. The inverse task is discussed, specifying the parameters of the output distributions and looking for the parameters of the input distributions. For larger standard deviations (stds) mixed Gaussian models are suggested as approximation of nonGaussian distributions.
\end{abstract}

Keywords: human-robot systems, navigation, Gaussian noise, Kalman filters, fuzzy modeling

\section{Introduction}

Human operators and mobile robots in shared work areas require a high degree of system stability, safety and mutual adaptation of the behavior to ensure a successful collaboration. A general discussion on robot-human cooperation is presented by [11]. Task planning, navigation and obstacle avoidance were major research activities in recent years $[9,3,14]$. Recognition of human intentions is a core issue for reaching particular goals $[19,4,12,13]$. The problem of intersection of trajectories between robots and humans is addressed by [2] while describing planned human-robot rendezvous at intersection areas. The computation of the intersections of the intended trajectories of robot and human requires the measurement and/or estimation of the the positions and orientations of robot and human which are subject to uncertainties and observation noise and thus affect 
the accuracy of the intersection estimates. [20] and [6] discuss a high level control strategy for a multiple target tracking approach for robots and other agents. On the other hand, the present work focuses on the one-robot one-human scenario to deepen the problem of accuracy and collision avoidance for short distances. Uncertainties of more than one degree in the orientation measurements on human and robot can lead to high uncertainties at intersection. For safety reasons and for an effective collaboration between humans and robots, it is therefore essential to predict uncertainties at possible intersections. Positions and orientations of human and robot are non-linearly related to the intersection coordinates but can be linearized if we only consider the linear part of correlation between input (positions and orientations) and output (intersection coordinates) and for small stds at the input $[16,1]$. The problem of uncertainty in human/robot systems leads us consequently to fuzzy systems due to the human factor involved and the issue of uncertain inputs in technical systems. We know two main directions to deal with uncertainties at system inputs:

- processing of fuzzy inputs (fuzzy sets) in fuzzy systems $[17,10,5]$.

- fuzzy reasoning with probabilistic inputs [21] and the transformation of probabilistic distributions into fuzzy sets [15].

Despite the success of these approaches in fuzzy systems, these methods fail to address the practical problem of processing a probability distribution through a static nonlinear system, which is described both analytically and fuzzy. Dealing with uncertain/fuzzy inputs in an analytical way is motivated by prediction of future situations like collision avoidance or cooperation at specific work areas, and to use this information for feed forward control actions and/or re-planning of trajectories. Our main application is the bearing task for intersections of possible trajectories of human and robot starting from different positions for the same "target" (the intersection)[18]. At first we consider the static case (human and robot standing still) in order to show the general problems and challenges. A next step is to consider Robot and human in motion where Kalman filters are used both for positions and orientations to suppress the measurement noise during motion. For larger stds at the input, we introduce mixed Gaussian distributions. In principle we address the following direct task: given the parameters of Gaussian distributions at the input of a static system (human/robot), find the corresponding distribution parameters at the output (intersection). The inverse task reads: Given the output distribution parameters, find the input distribution parameters. The paper is organized as follows. Section 2 describes the analytical way to the intersection problem with Gaussian noise. Section 3 deals with the inverse problem to find the input distribution parameters for given output parameters. Section 4 describes the local linear fuzzy approximation of the nonlinear analytical calculation. In Section 5 the two orientation inputs are extended by another four position inputs, a so-called "energy" approach is introduced to solve the overdetermined problem: 6 inputs and 2 outputs In Section 6, mixed Gaussian distributions and their contribution to the intersection problem are presented. Section 7 deals with robot and human in motion with Kalman filters for positions and orientations included.Section 8 deals with simulations to eval- 
uate the methods and approaches presented. Finally, Section 9 concludes the paper.

\section{Gaussian noise and the intersection problem}

\subsection{Computation of intersections - analytical approach}

Consider two linear paths $\mathbf{x}_{R}(t)$ and $\mathbf{x}_{H}(t)$ intended by a robot and a human and a possible intersection $\left(x_{c}, y_{c}\right) \cdot \mathbf{x}_{H}=\left(x_{H}, y_{H}\right)$ and $\mathbf{x}_{R}=\left(x_{R}, y_{R}\right)$ are the position of human and robot and $\phi_{H}$ and $\phi_{R}$ their orientation angles (see Fig. $1)$.

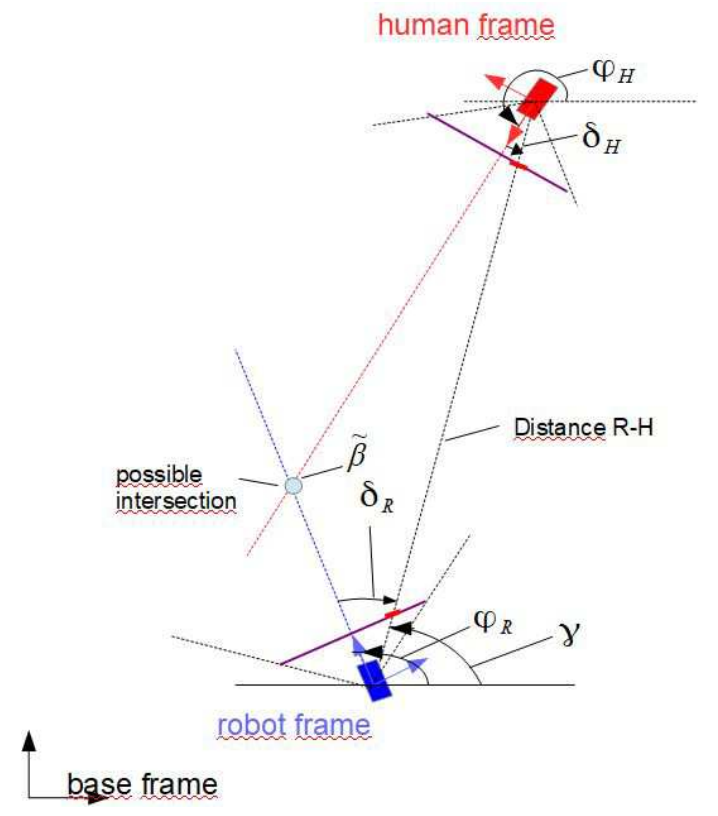

Fig. 1. Human-robot scenario: geometry, extracted from [18]

From Fig.1 we get

$$
\begin{array}{r}
x_{H}=x_{R}+d_{R H} \cos \left(\phi_{R}+\delta_{R}\right) \\
y_{H}=y_{R}+d_{R H} \sin \left(\phi_{R}+\delta_{R}\right) \\
x_{R}=x_{H}+d_{R H} \cos \left(\phi_{H}+\delta_{H}\right) \\
y_{R}=y_{H}+d_{R H} \sin \left(\phi_{H}+\delta_{H}\right)
\end{array}
$$

where positive angles $\delta_{H}$ and $\delta_{R}$ are measured from the $y$ coordinates counterclockwise and $\mathbf{x}_{H}, \mathbf{x}_{R}, \phi_{R}, \delta_{H}, \delta_{R}, d_{R H}$ and the angle $\gamma$ are supposed to be 
measurable. The orientation angle $\phi_{H}$ is computed by

$$
\phi_{H}=\arcsin \left(\left(y_{H}-y_{R}\right) / d_{R H}\right)-\delta_{H}+\pi
$$

From fig. 1, equations (1) and (2) the intersection coordinates $x_{c}$ and $y_{c}$ are computed by

$$
\begin{aligned}
x_{c} & =\frac{A-B}{\tan \phi_{R}-\tan \phi_{H}} \\
y_{c} & =\frac{A \tan \phi_{H}-B \tan \phi_{R}}{\tan \phi_{R}-\tan \phi_{H}} \\
A & =x_{R} \tan \phi_{R}-y_{R} \\
B & =x_{H} \tan \phi_{H}-y_{H}
\end{aligned}
$$

Rewriting (3) into a matrix-vector form leads to

$$
\mathbf{x}_{c}=\left(x_{c}, y_{c}\right)^{T} \text { and } \mathbf{x}_{R H}=\left(x_{R}, y_{R}, x_{H}, y_{H}\right)^{T}
$$

$$
\mathbf{x}_{c}=A_{R H} \cdot \mathbf{x}_{R H}
$$

where $\mathbf{x}_{c}=\left(x_{c}, y_{c}\right)^{T}$ and $\mathbf{x}_{R H}=\left(x_{R}, y_{R}, x_{H}, y_{H}\right)^{T}$ and

$$
\begin{aligned}
& A_{R H}=f\left(\phi_{R}, \phi_{H}\right)= \\
& \frac{1}{G}\left(\begin{array}{cccc}
\tan \phi_{R} & -1 & -\tan \phi_{H} & 1 \\
\tan \phi_{R} \tan \phi_{H} & -\tan \phi_{H}-\tan \phi_{R} \tan \phi_{H} \tan \phi_{H}
\end{array}\right) ; G=\tan \phi_{R}-\tan \phi_{H}
\end{aligned}
$$

The orientation angle $\phi_{H}$ can be determined by different means, for example from a scenario recorded by human eye tracking plus a corresponding camera picture taken from the human's position and transmitted to the robot [14].

A Takagi-Sugeno (TS) fuzzy approximation of (4) is derived by [14]

$$
\mathbf{x}_{c}=\sum_{i, j} w_{i}\left(\phi_{R}\right) w_{j}\left(\phi_{H}\right) \cdot A_{R H i, j} \cdot \mathbf{x}_{R H}
$$

$w_{i}\left(\phi_{R}\right), w_{j}\left(\phi_{H}\right) \in[0,1]$ are normalized membership functions with $\sum_{i} w_{i}\left(\phi_{R}\right)=$ 1 and $\sum_{j} w_{j}\left(\phi_{H}\right)=1$. In the following paragraph the accuracy of the computed intersection in the case of distorted orientation information is addressed.

\subsection{Transformation of Gaussian distributions}

General considerations Let be given a static nonlinear system

$$
\mathbf{z}=F(\mathbf{x})
$$

with two inputs $\mathbf{x}=\left(x_{1}, x_{2}\right)^{T}$ and two outputs $\mathbf{z}=\left(z_{1}, z_{2}\right)^{T}$ where $F$ denotes a nonlinear system. Furthermore let two uncorrelated Gaussian distributed inputs $x_{1}$ and $x_{2}$ be described by the 2-dim density 


$$
f_{x_{1}, x_{2}}=\frac{1}{2 \pi \sigma_{x_{1}} \sigma_{x_{2}}} \exp \left(-\frac{1}{2}\left(\frac{e_{x_{1}}^{2}}{\sigma_{x_{1}}^{2}}+\frac{e_{x_{2}}^{2}}{\sigma_{x_{2}}^{2}}\right)\right)
$$

where $e_{x_{1}}=x_{1}-\overline{x_{1}}, \overline{x_{1}}-\operatorname{mean}\left(x_{1}\right), \sigma_{x_{1}}$ - standard deviation $x_{1}$ and $e_{x_{2}}=$ $x_{2}-\overline{x_{2}}, \overline{x_{2}}-\operatorname{mean}\left(x_{2}\right), \sigma_{x_{2}}$ - standard deviation $x_{2}$.

The goal is to find the distribution of the output signals $z_{1}$ and $z_{2}$ and thus their stds and the correlation coefficient between them. For linear systems Gaussian distributions are linearly transformed so that the output signals are also Gaussian distributed. However this does not hold for nonlinear system in general. Only if we assume the input stds small enough then the output distributions are nearly Gaussian distributed but with correlated components as follows

$$
\begin{aligned}
& f_{z_{1}, z_{2}}=\frac{1}{2 \pi \sigma_{z_{1}} \sigma_{z_{2}} \sqrt{1-\rho_{z_{12}}^{2}}} \cdot \\
& \quad \exp \left(-\frac{1}{2\left(1-\rho_{z_{12}}^{2}\right)}\left(\frac{e_{z_{1}}^{2}}{\sigma_{z_{1}}^{2}}+\frac{e_{z_{2}}^{2}}{\sigma_{z_{2}}^{2}}-\frac{2 \rho_{z_{12}} e_{z_{1}} e_{z_{2}}}{\sigma_{z_{1}} \sigma_{z_{2}}}\right)\right)
\end{aligned}
$$

$\rho_{z_{12}}$ - correlation coefficient. For the connection between (7) and (8) we use a differential approach which is described in the next paragraph.

Differential approach Function F in (6) can be described by individual smooth and nonlinear static transfer functions where $\left(x_{1}, x_{2}\right)=\left(\phi_{R}, \phi_{H}\right)$ and $\left(z_{1}, z_{2}\right)=$ $\left(x_{c}, y_{c}\right)$

$$
\begin{aligned}
& z_{1}=f_{1}\left(x_{1}, x_{2}\right) \\
& z_{2}=f_{2}\left(x_{1}, x_{2}\right)
\end{aligned}
$$

Linearization of (9) yields

$$
\mathbf{d z}=\tilde{J} \cdot \mathbf{d} \mathbf{x} \quad \text { or } \quad \mathbf{e}_{\mathbf{z}}=\tilde{J} \cdot \mathbf{e}_{\mathbf{x}}
$$

with

$$
\begin{gathered}
\mathbf{e}_{\mathbf{z}}=\left(e_{z_{1}}, e_{z_{2}}\right)^{T} \quad \text { and } \quad \mathbf{e}_{\mathbf{x}}=\left(e_{x_{1}}, e_{x_{2}}\right)^{T} \\
\mathbf{d z}=\left(d z_{1}, d z_{2}\right)^{T} \quad \text { and } \quad \mathbf{d x}=\left(d x_{1}, d x_{2}\right)^{T} \\
\tilde{J}=\left(\begin{array}{c}
\partial f_{1} / \partial x_{1}, \partial f_{1} / \partial x_{2} \\
\partial f_{2} / \partial x_{1}, \partial f_{2} / \partial x_{2}
\end{array}\right)
\end{gathered}
$$


Specific approach to the intersection From (4) we derive the differential approach if the contributing agents change their directions of motion. To quantify the uncertainty of $\mathbf{x}_{c}$ for uncertain angles $\phi_{R}$ and $\phi_{H}$ or positions $\mathbf{x}_{R H}=\left(x_{R}, y_{R}, x_{H}, y_{H}\right)^{T}$ we differentiate (4) with $\mathbf{x}_{R H}=$ const.

$$
\begin{gathered}
\dot{\mathbf{x}}_{c}=\tilde{J} \cdot \dot{\phi} \\
\dot{\phi}=\left(\begin{array}{lll}
\dot{\phi}_{R} & \dot{\phi}_{H}
\end{array}\right)^{T} ; \quad \tilde{J}=\left(\begin{array}{cc}
\tilde{J}_{11} & \tilde{J}_{12} \\
\tilde{J}_{21} & \tilde{J}_{22}
\end{array}\right)
\end{gathered}
$$

where

$$
\begin{aligned}
\tilde{J}_{11} & =\left(-\tan \phi_{H} 1 \tan \phi_{H}-1\right) \frac{\mathbf{x}_{R H}}{G^{2} \cdot \cos ^{2} \phi_{R}} \\
\tilde{J}_{12} & =\left(\tan \phi_{R}-1-\tan \phi_{R} 1\right) \frac{\mathbf{x}_{R H}}{G^{2} \cdot \cos ^{2} \phi_{H}} \\
\tilde{J}_{21} & =\tilde{J}_{11} \cdot \tan \phi_{H} \\
\tilde{J}_{22} & =\tilde{J}_{12} \cdot \tan \phi_{R}
\end{aligned}
$$

Output distribution To compute the density $f_{z_{1}, z_{2}}$ of the output signal we invert (11) and substitute the entries of $\mathbf{e}_{\mathbf{x}}$ into (7)

$$
\mathbf{e}_{\mathbf{x}}=J \cdot \mathbf{e}_{\mathbf{z}}
$$

with $J=\tilde{J}^{-1}$ and

$$
J=\left(\begin{array}{ll}
J_{11} & J_{12} \\
J_{21} & J_{22}
\end{array}\right)=\left(\begin{array}{l}
\mathbf{j}_{\mathbf{x z}} \\
\mathbf{j}_{\mathbf{y z}}
\end{array}\right)
$$

where $\mathbf{j}_{\mathbf{x z}}=\left(J_{11}, J_{12}\right)$ and $\mathbf{j}_{\mathbf{y z}}=\left(J_{21}, J_{22}\right)$. The entries $J_{i j}$ are the result of the inversion of $\tilde{J}$. From this substitution we get

$$
f_{x_{1}, x_{2}}=K_{x_{1}, x_{2}} \cdot \exp \left(-\frac{1}{2} \cdot \mathbf{e}_{\mathbf{z}}^{T} \cdot\left(\mathbf{j}_{\mathbf{x}_{1}, \mathbf{z}}^{T}, \mathbf{j}_{\mathbf{x}_{\mathbf{2}}, \mathbf{z}}{ }^{T}\right) \cdot S_{x}^{-1} \cdot\left(\begin{array}{l}
\mathbf{j}_{\mathbf{x}_{1}, \mathbf{z}} \\
\mathbf{j}_{\mathbf{x}_{\mathbf{2}}, \mathbf{z}}
\end{array}\right) \cdot \mathbf{e}_{\mathbf{z}}\right)
$$

where $K_{x_{1}, x_{2}}=\frac{1}{2 \pi \sigma_{x_{1}} \sigma_{x_{2}}}$ and

$$
S_{x}^{-1}=\left(\begin{array}{c}
\frac{1}{\sigma_{x_{1}}^{2}}, 0 \\
0, \frac{1}{\sigma_{x_{2}}^{2}}
\end{array}\right)
$$

The exponent of (16) is rewritten into 


$$
x p o=-\frac{1}{2} \cdot\left(\frac{1}{\sigma_{x_{1}}^{2}}\left(e_{z_{1}} J_{11}+e_{z_{2}} J_{12}\right)^{2}+\frac{1}{\sigma_{x_{2}}^{2}}\left(e_{z_{1}} J_{21}+e_{z_{2}} J_{22}\right)^{2}\right)
$$

and furthermore

$$
x p o=-\frac{1}{2} \cdot\left[e_{z_{1}}^{2}\left(\frac{J_{11}^{2}}{\sigma_{x_{1}}^{2}}+\frac{J_{21}^{2}}{\sigma_{x_{2}}^{2}}\right)+e_{z_{2}}^{2}\left(\frac{J_{12}^{2}}{\sigma_{x_{1}}^{2}}+\frac{J_{22}^{2}}{\sigma_{x_{2}}^{2}}\right)+2 \cdot e_{z_{1}} e_{z_{2}}\left(\frac{J_{11} J_{12}}{\sigma_{x_{1}}^{2}}+\frac{J_{21} J_{22}}{\sigma_{x_{2}}^{2}}\right)\right]
$$

Then, we compare xpo in (19) with the exponent of the output density (8) Let

$$
A=\left(\frac{J_{11}^{2}}{\sigma_{x_{1}}^{2}}+\frac{J_{21}^{2}}{\sigma_{x_{2}}^{2}}\right) ; \quad B=\left(\frac{J_{12}^{2}}{\sigma_{x_{1}}^{2}}+\frac{J_{22}^{2}}{\sigma_{x_{2}}^{2}}\right) ; \quad C=\left(\frac{J_{11} J_{12}}{\sigma_{x_{1}}^{2}}+\frac{J_{21} J_{22}}{\sigma_{x_{2}}^{2}}\right)
$$

then this comparison yields

$$
\frac{1}{\left(1-\rho_{z_{12}}^{2}\right)} \frac{1}{\sigma_{z_{1}}^{2}}=A ; \quad \frac{1}{\left(1-\rho_{z_{12}}^{2}\right)} \frac{1}{\sigma_{z_{2}}^{2}}=B ; \quad \frac{-2 \rho_{z_{12}}}{\left(1-\rho_{z_{12}}^{2}\right)} \frac{1}{\sigma_{z_{1}} \sigma_{z_{2}}}=2 C
$$

from which we finally obtain the correlation coefficient $\rho_{z_{12}}$ and the stds $\sigma_{z_{1}}$ and $\sigma_{z_{2}}$

$$
\rho_{z_{12}}=-\frac{C}{\sqrt{A B}} ; \quad \frac{1}{\sigma_{z_{1}}^{2}}=A-\frac{C^{2}}{B} ; \quad \frac{1}{\sigma_{z_{2}}^{2}}=B-\frac{C^{2}}{A}
$$

from which yields: once we have measured the parameters of the input distribution and the mathematical expression for the transfer function $F(x, y)$ then we can compute the output distribution parameters directly.

\section{Inverse Solution}

Up to now we discussed the problem: Given the parameters of the input distributions of a nonlinear system, find the parameters of the output distributions. In practice, it might be helpful to define a specific accuracy at the intersection and look for the necessary accuracy of the input measurements.

This inverse task we apply is similar to that we discussed in section 2.2. The starting point is equation (11). Equations (7) and (8) describe the densities of the inputs and the outputs, respectively. Then we substitute (11) into (8) and discuss the exponent $x p o_{z}$ only:

$$
x p o_{z}=\frac{-1}{2\left(1-\rho_{z_{12}}^{2}\right)}\left(\mathbf{e}_{\mathbf{x}}^{T} \tilde{J}^{T} S_{z}^{-1} \tilde{J} \mathbf{e}_{\mathbf{x}}-\frac{2 \rho_{z_{12}} e_{z_{1}} e_{z_{2}}}{\sigma_{z_{1}} \sigma_{z_{2}}}\right)
$$

where

$$
S_{z}^{-1}=\left(\begin{array}{c}
\frac{1}{\sigma_{z_{1}}^{2}}, 0 \\
0, \frac{1}{\sigma_{z_{1}}^{2}}
\end{array}\right)
$$


With

$$
\begin{array}{r}
e_{z_{1}} e_{z_{2}}=\left(\tilde{J}_{11} e_{x_{1}}+\tilde{J}_{12} e_{x_{2}}\right) \cdot\left(\tilde{J}_{21} e_{x_{1}}+\tilde{J}_{22} e_{x_{2}}\right) ; \\
\mathbf{e}_{\mathbf{x}}^{T} \tilde{J}^{T} S_{z}^{-1} \tilde{J} \mathbf{e}_{\mathbf{x}}=e_{x_{1}}^{2}\left(\frac{\tilde{J}_{11}^{2}}{\sigma_{z_{1}}^{2}}+\frac{\tilde{J}_{21}^{2}}{\sigma_{z_{2}}^{2}}\right)+e_{x_{2}}^{2}\left(\frac{\tilde{J}_{12}^{2}}{\sigma_{z_{1}}^{2}}+\frac{\tilde{J}_{22}^{2}}{\sigma_{z_{2}}^{2}}\right) \\
+2 e_{x_{1}} e_{x_{2}}\left(\frac{\tilde{J}_{11} \tilde{J}_{12}}{\sigma_{z_{1}}^{2}}+\frac{\tilde{J}_{21} \tilde{J}_{22}}{\sigma_{z_{2}}^{2}}\right)
\end{array}
$$

while renaming we obtain $x p o_{z}$ into $x p o_{x}$ we obtain

$$
\begin{array}{r}
x p o_{z}=-\frac{1}{2}\left(e_{x_{1}}^{2}\left(\frac{\tilde{J}_{11}^{2}}{\sigma_{z_{1}}^{2}}+\frac{\tilde{J}_{21}^{2}}{\sigma_{z_{2}}^{2}}-\frac{2 \rho_{z_{12}}}{\sigma_{z_{1}} \sigma_{z_{2}}} \tilde{J}_{11} \tilde{J}_{21}\right) /\left(1-\rho_{z_{12}}^{2}\right)\right. \\
+e_{x_{2}}^{2}\left(\frac{\tilde{J}_{12}^{2}}{\sigma_{z_{1}}^{2}}+\frac{\tilde{J}_{22}^{2}}{\sigma_{z_{2}}^{2}}-\frac{2 \rho_{z_{12}}}{\sigma_{z_{1}} \sigma_{z_{2}}} \tilde{J}_{12} \tilde{J}_{22}\right) /\left(1-\rho_{z_{12}}^{2}\right) \\
\left.+\frac{2 e_{x_{1}} e_{x_{2}}}{\left(1-\rho_{z_{12}}^{2}\right)} \cdot\left(\frac{\tilde{J}_{11} \tilde{J}_{12}}{\sigma_{z_{1}}^{2}}+\frac{\tilde{J}_{21}}{\sigma_{z_{2}}^{2}}-\frac{\tilde{J}_{22}}{\sigma_{z_{1}} \sigma_{z_{2}}}\left(\tilde{J}_{11} \tilde{J}_{22}+\tilde{J}_{12} \tilde{J}_{21}\right)\right)\right)
\end{array}
$$

Now, comparing (26) with the exponent of (7) of the input density we find that the mixed term in (26) should be zero. Hence we obtain the correlation coefficient and the stds of the inputs as follows

$$
\begin{gathered}
\rho_{z_{12}}=\left(\frac{\tilde{J}_{11} \tilde{J}_{12}}{\sigma_{z_{1}}^{2}}+\frac{\tilde{J}_{21} \tilde{J}_{22}}{\sigma_{z_{2}}^{2}}\right) \frac{\sigma_{z_{1}} \sigma_{z_{2}}}{\left(\tilde{J}_{11} \tilde{J}_{22}+\tilde{J}_{12} \tilde{J}_{21}\right)} \\
\frac{1}{\sigma_{x}^{2}}=\left(\frac{\tilde{J}_{11}^{2}}{\sigma_{z_{1}}^{2}}+\frac{\tilde{J}_{21}^{2}}{\sigma_{z_{2}}^{2}}-\frac{2 \rho_{z_{12}}}{\sigma_{z_{1}} \sigma_{z_{2}}} \tilde{J}_{11} \tilde{J}_{21}\right) /\left(1-\rho_{z_{12}}^{2}\right) \\
\frac{1}{\sigma_{y}^{2}}=\left(\frac{\tilde{J}_{12}^{2}}{\sigma_{z_{1}}^{2}}+\frac{\tilde{J}_{22}^{2}}{\sigma_{z_{2}}^{2}}-\frac{2 \rho_{z_{12}}}{\sigma_{z_{1}} \sigma_{z_{2}}} \tilde{J}_{12} \tilde{J}_{22}\right) /\left(1-\rho_{z_{12}}^{2}\right)
\end{gathered}
$$

\section{FUZZY SOLUTION}

To avoid high costs of an on-line computation of the output distribution a TSfuzzy approximation of (26) is suggested by the following rules $R_{i j}$ provided that an analytical representation (6) is available

$$
\begin{array}{rcccc}
R_{i j}: I F \quad x_{1}=X_{1 i} & A N D \quad x_{2}=X_{2 i} & \text { THEN } & \rho_{z_{12}}=-\frac{C_{i j}}{\sqrt{A_{i j} B_{i j}}} \\
& A N D \quad \frac{1}{\sigma_{z_{1}}^{2}}=A_{i j}-\frac{C_{i j}^{2}}{B_{i j}} & A N D & \frac{1}{\sigma_{z_{2}}^{2}}=B_{i j}-\frac{C_{i j}^{2}}{A_{i j}}
\end{array}
$$

where $X_{1 i}, X_{2 i}$ are fuzzy terms for $x_{1}, x_{2}, A_{i j}, B_{i j}, C_{i j}$ are functions of predefined variables $x_{1}=x_{1 i}$ and $x_{2}=x_{2 i}$ 
From (30) we get

$$
\begin{aligned}
\rho_{z_{12}} & =-\sum_{i j} w_{i}\left(x_{1}\right) w_{j}\left(x_{2}\right) \frac{C_{i j}}{\sqrt{A_{i j} B_{i j}}} \\
\frac{1}{\sigma_{z_{1}}^{2}} & =\sum_{i j} w_{i}\left(x_{1}\right) w_{j}\left(x_{2}\right)\left(A_{i j}-\frac{C_{i j}^{2}}{B_{i j}}\right) \\
\frac{1}{\sigma_{z_{2}}^{2}} & =\sum_{i j} w_{i}\left(x_{1}\right) w_{j}\left(x_{2}\right)\left(B_{i j}-\frac{C_{i j}^{2}}{A_{i j}}\right)
\end{aligned}
$$

$w_{i}\left(x_{1}\right) \in[0,1]$ and $w_{j}\left(x_{2}\right) \in[0,1]$ are weighting functions with $\sum_{i} w_{i}\left(x_{1}\right)=1$ $\sum_{j} w_{j}\left(x_{2}\right)=1$

\section{EXTENSION TO SIX INPUTS AND TWO OUTPUTS}

\subsection{General approach}

In the previous section we dealt with two orientation inputs and two intersection position outputs where the position coordinates of robot and human are assumed to be constant.

Consider again the nonlinear system

$$
\mathbf{x}_{\mathbf{c}}=F(\mathbf{x})
$$

where $F$ denotes a nonlinear system.

Here we have 6 inputs $\mathbf{x}=\left(x_{1}, x_{2}, x_{3}, x_{4}, x_{5}, x_{6}\right)^{T}$ and 2 outputs $\mathbf{x}_{\mathbf{c}}=\left(x_{c}, y_{c}\right)^{T}$. For the intersection problem we get $\mathbf{x}=\left(\phi_{R}, \phi_{H}, x_{R}, y_{R}, x_{H}, y_{H}\right)$ Let further the uncorrelated Gaussian distributed inputs $x_{1} \ldots x_{6}$ be described by the 6 -dim density

$$
f_{x_{i}}=\frac{1}{(2 \pi)^{6 / 2}\left|S_{x}\right|^{1 / 2}} \exp \left(-\frac{1}{2}\left(\mathbf{e}_{\mathbf{x}}^{T} S_{x}^{-1} \mathbf{e}_{\mathbf{x}}\right)\right)
$$

where $\mathbf{e}_{\mathbf{x}}=\left(e_{x 1}, e_{x 2}, \ldots, e_{x 6}\right)^{T} ; \mathbf{e}_{\mathbf{x}}=\mathbf{x}-\overline{\mathbf{x}}, \overline{\mathbf{x}}$ - mean $(\mathbf{x}), S_{x}$ - covariance matrix.

$$
S_{x}=\left(\begin{array}{cccc}
\sigma_{x_{1}}^{2} & 0 & \ldots & 0 \\
0 & \sigma_{x_{2}}^{2} & \ldots & 0 \\
\ldots & \ldots & \ldots & \ldots \\
0 & \ldots & 0 & \sigma_{x_{6}}^{2}
\end{array}\right)
$$

The output density is again described by

$$
f_{x_{c}, y_{c}}=\frac{1}{2 \pi \sigma_{x_{c}} \sigma_{y_{c}} \sqrt{1-\rho^{2}}} \cdot \exp \left(-\frac{1}{2\left(1-\rho^{2}\right)}\left(\mathbf{e}_{\mathbf{x}_{\mathbf{c}}}^{\mathbf{T}} S_{c}{ }^{-1} \mathbf{e}_{\mathbf{x}_{\mathbf{c}}}-\frac{2 \rho e_{x_{c}} e_{y_{c}}}{\sigma_{x_{c}} \sigma_{y_{c}}}\right)\right)
$$


where $\rho$ - correlation coefficient, $\mathbf{e}_{\mathbf{x}_{\mathbf{c}}}=\left(e_{x_{c}}, e_{y_{c}}\right)^{T}$ and

$$
S_{c}{ }^{-1}=\left(\begin{array}{l}
\frac{1}{\sigma_{x_{c}}^{2}}, 0 \\
0, \frac{1}{\sigma_{y_{c}}^{2}}
\end{array}\right)
$$

In correspondence to (6) and (9) function $\mathrm{F}$ can be described by

$$
\begin{aligned}
& x_{c}=f_{1}(\mathbf{x}) \\
& y_{c}=f_{2}(\mathbf{x})
\end{aligned}
$$

Furthermore according to (13) we have

$$
\mathbf{e}_{\mathbf{x}_{c}}=\tilde{J} \cdot \mathbf{e}_{\mathbf{x}}
$$

with

$$
\tilde{J}=\left(\begin{array}{llll}
\tilde{J}_{11} & \tilde{J}_{12} & \ldots & \tilde{J}_{16} \\
\tilde{J}_{21} & \tilde{J}_{22} & \ldots & \tilde{J}_{26}
\end{array}\right)
$$

where

$$
\tilde{J}_{i j}=\frac{\partial f_{i}}{\partial x_{j}}, \quad, i=1,2 \quad, j=1, \ldots, 6
$$

Inversion of (38) leads to

$$
\mathbf{e}_{\mathbf{x}}=\tilde{J}^{t} \cdot \mathbf{e}_{\mathbf{x}_{\mathbf{c}}}=J \cdot \mathbf{e}_{\mathbf{x}_{\mathbf{c}}}
$$

with the pseudo inverse $\tilde{J}^{t}$ of $\tilde{J}$. Renaming $\tilde{J}^{t}$ into $J$ we get

$$
J=\left(\begin{array}{cc}
J_{11} & J_{12} \\
\ldots & \ldots \\
J_{61} & J_{62}
\end{array}\right)
$$

Substituting (37) into (33) we obtain

$$
f_{x_{c}, y_{c}}=K_{x_{c}} \exp \left(-\frac{1}{2}\left(\mathbf{e}_{\mathbf{x}_{\mathbf{c}}}{ }^{T} J^{T} S_{x}{ }^{-1} J \mathbf{e}_{\mathbf{x}_{\mathbf{c}}}\right)\right)
$$

where $K_{x_{c}}$ represents a normalization of the output density and

$$
J_{x_{c}}=J^{T} S_{x}{ }^{-1} J=\left(\begin{array}{ll}
A & B \\
C & D
\end{array}\right)
$$


where

$$
\begin{aligned}
& A=\sum_{i=1}^{6} \frac{1}{\sigma_{x_{i}}^{2}} J_{i 1}^{2} ; \quad B=\sum_{i=1}^{6} \frac{1}{\sigma_{x_{i}}^{2}} J_{i 1} J_{i 2} \\
& C=\sum_{i=1}^{6} \frac{1}{\sigma_{x_{i}}^{2}} J_{i 1} J_{i 2} ; \quad D=\sum_{i=1}^{6} \frac{1}{\sigma_{x_{i}}^{2}} J_{i 2}^{2}
\end{aligned}
$$

Substitution of (43) into (42) leads with $B=C$ to

$$
f_{x_{c}, y_{c}}=K_{x_{c}} \exp \left(-\frac{1}{2}\left(A e_{x_{c}}^{2}+D e_{y_{c}}^{2}+2 C e_{x_{c}} e_{y_{c}}\right)\right)
$$

Comparison of (44) with (34) leads with (35) to

$$
\begin{aligned}
\rho & =-\frac{C}{\sqrt{A D}} \\
\frac{1}{\sigma_{x_{c}}^{2}} & =A-\frac{C^{2}}{D} ; \quad \frac{1}{\sigma_{y_{c}}^{2}}=D-\frac{C^{2}}{A}
\end{aligned}
$$

which is the counterpart to the 2-dim input case (22).

\subsection{Fuzzy approach}

The fuzzy approach is similar to the 2-input 2-output case: The first step is to compute values $A_{i}, B_{i}$ and $C_{i}$ from (43) at predefined positions/orientations $\mathbf{x}=\left(x_{1}, x_{2}, x_{3}, x_{4}, x_{5}, x_{6}\right)_{i}^{T}$. Then, we formulate fuzzy rules $R_{i}$, according to (30) and (31) with $i=1 \ldots n, l$ - number of fuzzy terms, $k=6$ - number of variables $n=l^{k}$ - number of rules.

$$
\begin{gathered}
R_{i}: \quad I F \quad \mathbf{x}_{i}=\mathbf{X}_{i} \quad \text { THEN } \quad \rho_{z_{12}}=-\frac{C_{i}}{\sqrt{A_{i} B_{i}}} \\
A N D \quad \frac{1}{\sigma_{z_{1}}^{2}}=A_{i}-\frac{C_{i}^{2}}{B_{i}} \quad \text { AND } \quad \frac{1}{\sigma_{z_{2}}^{2}}=B_{i}-\frac{C_{i}^{2}}{A_{i}}
\end{gathered}
$$

where $\mathbf{X}_{i}$ are fuzzy terms for $\mathbf{x}_{i}$. From this set of rules we get again (31).

$$
\begin{gathered}
\rho_{z_{12}}=-\sum_{i} w_{i}(\mathbf{x}) \frac{C_{i}}{\sqrt{A_{i} B_{i}}} \\
\frac{1}{\sigma_{z_{1}}^{2}}=\sum_{i} w_{i}(\mathbf{x})\left(A_{i}-\frac{C_{i}^{2}}{B_{i}}\right) \\
\frac{1}{\sigma_{z_{2}}^{2}}=\sum_{i} w_{i}(\mathbf{x})\left(B_{i}-\frac{C_{i}^{2}}{A_{i}}\right)
\end{gathered}
$$

$w_{i}(\mathbf{x})=\Pi_{l=1}^{6} w_{i}\left(x_{l}\right), w_{i}\left(x_{l}\right) \in[0,1]$ are weighting functions with $\sum_{i} w_{i}\left(x_{l}\right)=1$ 
The challenge of this approach is the number of rules needed. Even for the 2 -inputs 2-outputs case, the increase of the resolution for the same range of $\phi_{R}$ and $\phi_{H}$ yields an increase of the number of rules. Using 7 membership functions for $\phi_{R}$ and $\phi_{H}$ each we obtain 49 rules. Doubling of the number of membership functions leads to to 196 rules. To avoid an "explosion" of the number of rules to be processed at the same time a number of sub-areas together with a small set of rules is formulated. Then, depending on measurements of $\phi_{R}$ and $\phi_{H}$, an appropriate sub-area is selected, and the corresponding set of rules will be activated (see Fig. 2, sub-area $A_{R}, A_{H}$ ). So, the total number of rules could increase whereas the number of rules to be processed for a calculation would remain low. To avoid abrupt changes at the borderlines between the sub-areas an overlap of these regions is recommended. Unfortunately, for 6 inputs one faces an exponential increase in the number of rules being associated with a very high computational burden. For $l=7$ fuzzy terms for each input variable $x_{k}, k=6$ we end up with $n=7^{6}$ rules which is much to high. A limitation to an appropriate number of variables at the input of a fuzzy system can be either heuristic or systematic to find out the most influential input variables [8] (see Fig.2).

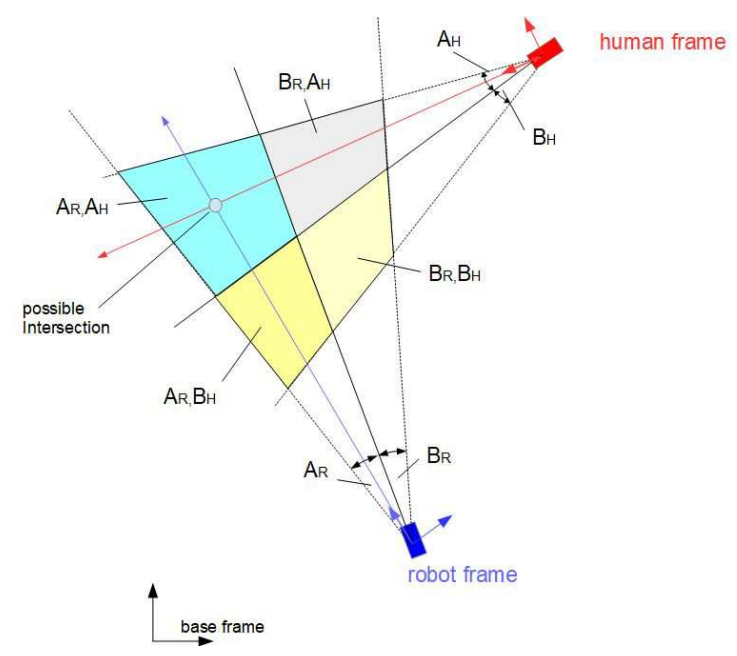

Fig. 2. Fuzzy sectors, extracted from [14]

\subsection{The energetic approach}

Simulations have shown that this method works well for certain conditions regarding the stds of orientations and positions of robot and human. However it appears that a mixture of orientation angles and robot/human positions leads to inconsistent results for stds $\sigma_{x_{c}}$ and $\sigma_{y_{c}}$ of the intersection coordinates $\mathbf{x}_{\mathbf{c}}$. The reason is the use of the pseudo inverse for the transformation $\mathbf{e}_{\mathbf{x}}=\tilde{J}^{t} \cdot \mathbf{e}_{\mathbf{x}_{\mathbf{c}}}$ in 
(40), a least square approximation, which leads definitely to uncertainties. A way out is to compute the variations of the intersection position from robot/human orientations $\mathbf{x}_{\mathbf{c} \phi \text {,tot }}$ and from robot/human positions $\mathbf{x}_{\mathbf{c} R H, t o t}$ separately

$$
\begin{array}{r}
\sigma_{\mathbf{x}_{\mathbf{c} \phi, t o t}}{ }^{2}=\sigma_{x_{c \phi}}{ }^{2}+\sigma_{y_{c \phi}}{ }^{2} \\
\sigma_{\mathbf{x}_{\mathbf{c}} R H, t o t}{ }^{2}=\sigma_{x_{c} R H}{ }^{2}+\sigma_{y_{c} R H}{ }^{2}
\end{array}
$$

Then both variations are summarized to

$$
\sigma_{\mathbf{x}_{\mathbf{c}} t o t}^{2}=\sigma_{\mathbf{x}_{\mathbf{c}} \phi, t o t}{ }^{2}+\sigma_{\mathbf{x}_{\mathbf{c}} R H, t o t}{ }^{2}
$$

Here it should be observed that the variance of an noise error signal $\mathbf{e}_{\mathbf{x}_{\mathbf{c}}}$ represents the noise energy per sample [7]. Due to the law of preservation of energy, the energy of the 'input' signal $\mathbf{e}_{\mathbf{x}}$ should be the same as that of the output $\mathbf{e}_{\mathbf{x}_{\mathbf{c}}}$ because of a transformation $\mathbf{e}_{\mathbf{x}_{\mathbf{c}}}=\tilde{J} \cdot \mathbf{e}_{\mathbf{x}}$ by which energy is neither fed-in nor gone-away. Following this idea it is obvious to use the resulting standard deviation

$$
\sigma_{\mathbf{x}_{\mathbf{c} t o t}}=\sqrt{\sigma_{\mathbf{x}_{\mathbf{c} \phi, t o t}}{ }^{2}+\sigma_{\mathbf{x}_{\mathbf{c}} R H, t o t}{ }^{2}}
$$

as a measure for the uncertainty of the intersection coordinates in the case of given orientation/position uncertainties of robot and human. The input energy $E_{i n}$ is computed by the energy $E_{\phi}$ from the orientation angles and by $E_{R H}$ from the positions of robot and human

$$
E_{i n}=E_{\phi}+E_{R H}
$$

where

$$
E_{\phi}=r_{x_{R}}^{2} \cdot \sigma_{\phi_{R}}^{2}+r_{x_{H}}^{2} \cdot \sigma_{\phi_{H}}^{2}
$$

and $r_{x_{R}}$ - distance robot-intersection, $r_{x_{H}}$ - distance human-intersection. Further

$$
E_{R H}=\sigma_{x_{R}}^{2}+\sigma_{y_{R}}^{2}+\sigma_{x_{H}}^{2}+\sigma_{y_{H}}^{2}
$$

The average standard deviation of the input signal reads

$$
\sigma_{i n}=\sqrt{E_{\phi}+E_{R H}}
$$

Since

$$
\sigma_{i n}=\sigma_{\mathbf{x}_{\mathbf{c} t o t}}
$$

we know the 'output' average standard deviation just from the 'input' average standard deviation on the condition that we know the intersection coordinate $\mathrm{x}_{\mathbf{c}}$. 


\section{Mixed Gaussian distributions}

Gaussian input signals at nonlinear or fuzzy systems with large stds do not usually lead to Gaussian output signals. Therefore we approximate a distribution with a large standard deviation by several distributions with small stds. In this connection, fuzzy systems are linearized around the mean values of these distributions. The following analysis shows that the previous analytical approach and the fuzzy approximation also applies for mixed Gaussian distributions. Consider an example of a mixture of two distributions/densities $f_{x y 1}$ and $f_{x y 2}$

$$
\begin{aligned}
& f_{x y 1}=\frac{1}{2 \pi \sigma_{x_{1}} \sigma_{y 1}} \exp \left(-\frac{1}{2}\left(\frac{e_{x_{1}}^{2}}{\sigma_{x_{1}}^{2}}+\frac{e_{y 1}^{2}}{\sigma_{y 1}^{2}}\right)\right) \\
& f_{x y 2}=\frac{1}{2 \pi \sigma_{x_{2}} \sigma_{y 2}} \exp \left(-\frac{1}{2}\left(\frac{e_{x_{2}}^{2}}{\sigma_{x_{2}}^{2}}+\frac{e_{y 2}^{2}}{\sigma_{y 2}^{2}}\right)\right)
\end{aligned}
$$

that are linearly combined

$$
f_{x y}=a_{1} f_{x y 1}+a_{2} f_{x y 2}
$$

with $a_{i}>=0$ and $\sum_{i} a_{i}=1$ where $i=1,2$ and

$$
\begin{array}{r}
e_{x_{1}}=x_{1}-\bar{x}_{1} ; \quad e_{x_{2}}=x_{2}-\bar{x}_{2} \\
e_{y 1}=y_{1}-\bar{y}_{1} ; \quad e_{y 2}=y_{2}-\bar{y}_{2}
\end{array}
$$

$\bar{x}_{i}, \bar{y}_{i}$ are the mean values of $x_{i}, y_{i}$.

The partial outputs yield

$$
f_{z_{1}, z_{2}}^{i}=\frac{1}{2 \pi \sigma_{z_{1}}^{i} \sigma_{z_{2}}^{i} \sqrt{1-\rho^{i^{2}}}} \cdot \exp \left(-\frac{1}{2\left(1-\rho^{i^{2}}\right)}\left(\frac{e_{z_{1}}^{i}{ }^{2}}{{\sigma_{z_{1}}^{i}}^{2}}+\frac{e_{z_{2}}^{{ }^{2}}{ }^{2}}{\sigma_{z_{2}}^{i}{ }^{2}}-\frac{2 \rho^{i} e_{z_{1}}^{i} e_{z_{2}}^{i}}{\sigma_{z_{1}}^{i} \sigma_{z_{2}}^{i}}\right)\right)(59)
$$

$e_{z_{1}}^{i}=z_{1}-\bar{z}_{1}^{i} ; e_{z_{2}}^{i}=z_{2}-\bar{z}_{2}^{i} ; \rho^{i}$ - correlation coefficient.

From this we finally obtain the output distribution

$$
f_{z_{1}, z_{2}}=\sum_{i=1}^{2} a_{i} f_{z_{1}, z_{2}}^{i}
$$

The mixed output distribution $f_{z_{1}, z_{2}}$ are linear combination of partial output distributions $f_{z_{1}, z_{2}}^{i}$ resulting from the input distributions $f_{x, y}^{i}$. Given the mean $\bar{z}_{k}^{i}, k=1,2$ and variance $\sigma_{z_{k}}^{i}{ }^{2}$ of the partial output distributions $f_{z_{1}, z_{2}}^{i}$, then mean and variance of the mixed output distribution are 


$$
\begin{aligned}
\bar{z}_{k} & =\sum_{i=1}^{2} \bar{z}_{k}^{i} \\
\sigma_{z_{k}}{ }^{2} & =a_{1}\left(\sigma_{z_{k 1}}\right)^{2}+a_{2}\left(\sigma_{z_{k} 2}\right)^{2}+a_{1} a_{2}\left(\bar{z}_{1}-\bar{z}_{2}\right)^{2}
\end{aligned}
$$

from which we obtain the standard deviation $\sigma_{z_{k}}$ of the intersection straight forward.

\section{Robots and humans in motion}

The previous sections dealt with the situation where robot and human are at rest. For robot and human in motion there occur the following new aspects:

- characteristic of the trajectories (form of paths, velocities)

- change of intersection positions during motion

- filtering of the positions/orientations of robot and human

- change of the errors of positions/orientations during motion.

Without restriction of generality we assume robot and human to move on lines with constant velocities and orientations since every smooth trajectory can be approximated by piecewise linear trajectories with regarding constant but usually different velocities. Positions, orientations and their velocities are corrupted with system noise and measurement noise. Therefore an appropriate discrete Kalman filter method is applied. For robot/human we have 6 inputs $\mathbf{x}(k)=\left(x, y, \phi, v_{x}, v_{y}, \omega\right)^{T}$ each. Starting with the system equations

$$
\begin{array}{r}
\text { state equation: } \mathbf{x}(k)=\mathbf{A}(k-1) \cdot \mathbf{x}(k-1)+\mathbf{w}(k-1) \\
\text { measurement equation: } \mathbf{y}(k)=\mathbf{C}(k) \cdot \mathbf{x}(k)+\mathbf{v}(k) \\
\text { covariance matrix of system noise }: E\left(\mathbf{w w}^{T}\right)=\mathbf{Q} \\
\text { covariance matrix of measurement noise }: E\left(\mathbf{v v}^{T}\right)=\mathbf{R} \\
\text { old estimation }: \hat{\mathbf{x}}(k-1) \\
\text { error }: \mathbf{e}=\mathbf{x}-\hat{\mathbf{x}} \\
\text { old error covariance matrix }: \mathbf{P}(k-1)=E\left(\mathbf{e} \cdot \mathbf{e}^{T}\right)
\end{array}
$$

A - system matrix, $\mathbf{C}$ - output matrix, $\mathbf{w}$ - system noise, $\mathbf{v}$ - measurement noise. Next, the two steps 'prediction' and 'correction' of the Kalman filter algorithm follow:

\section{Prediction}

This step includes the extrapolation of the state $\mathbf{x}(k)$ based on the previous estimation $\hat{\mathbf{x}}(k-1)$

$$
\mathbf{x}^{\star}(k)=\mathbf{A}(k-1) \cdot \hat{\mathbf{x}}(k-1)
$$

Furthermore, an extrapolation of matrix $\mathbf{P}(k-1)$ follows

$$
\mathbf{P}^{\star}(k)=\mathbf{Q}(k-1)+\mathbf{A}(k-1) \mathbf{P}(k-1) \mathbf{A}(k-1)^{T}
$$




\section{Correction}

Computation of the Kalman filter gain $\mathbf{K}(k)$ based on $\mathbf{P}^{\star}(k)$

$$
\mathbf{K}(k)=\mathbf{P}^{\star}(k) \cdot \mathbf{C}(k) \cdot\left(\mathbf{C}(k) \mathbf{P}^{\star}(k) \mathbf{C}(k)^{T}+\mathbf{R}(k)\right)^{-1}
$$

Computation of the new error covariance matrix $\mathbf{P}(\mathrm{k})$ using the Kalman filter gain $\mathbf{K}(k)$ and $\mathbf{P}^{\star}(k)$

$$
\mathbf{P}(k)=(I-\mathbf{K}(k)) \cdot \mathbf{C}(k)) \cdot \mathbf{P}^{\star}(k)
$$

Finally we obtain the new estimate

$$
\hat{\mathbf{x}}(k)=\mathbf{x}^{\star}(k)+\mathbf{K}(k) \cdot\left(\mathbf{y}(k)-\mathbf{C}(k) \cdot \mathbf{x}^{\star}(k)\right)
$$

The discrete Kalman filter will now be applied to the trajectories $\mathbf{x}(k)$ of the robot and the human to see how the intersection position $\mathbf{x}_{\mathbf{c}}$ evolves. Both the noise of robot/human positions and the noise of orientations have an impact on the noise of the intersection. However changes in orientations due to noise have a greater influence on the intersection that that of the positions. Therefore an appropriate Kalman filter is definitely needed. In the connection the combination of positions and orientation angles in a common Kalman filter is of great advantage and will be used in our analysis. An evaluation of the quality of the filter is done by the measurement of the standard deviations of the noise at the intersection at different segments $T 1, T 2, \ldots$ of the trajectories which is necessary because of different distances of the positions of the acting agents (robot and human) to the possible intersection of their trajectories (see Fig. 3). The structure of a trajectory either of the robot and the human is described by eq.(62).

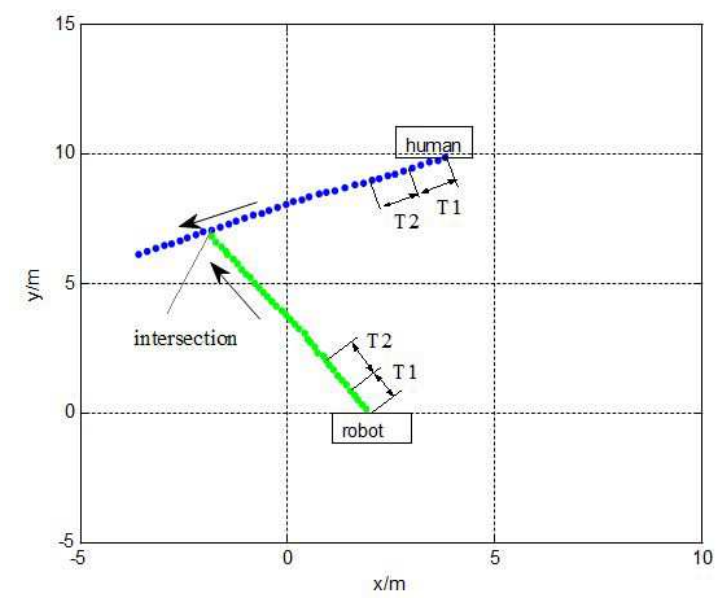

Fig. 3. Computation of the intersection during motion at different time sequences $\mathrm{Ti}$

With a discrete time step $\Delta t=1$ the corresponding matrices read 


$$
\begin{aligned}
& \mathbf{A}(\mathbf{k})=\left(\begin{array}{cccccc}
1 & 0 & 0 & \Delta t & 0 & 0 \\
0 & 1 & 0 & 0 & \Delta t & 0 \\
0 & 0 & 1 & 0 & 0 & \Delta t \\
0 & 0 & 0 & 1 & 0 & 0 \\
0 & 0 & 0 & 0 & 1 & 0 \\
0 & 0 & 0 & 0 & 0 & 1
\end{array}\right) ; \quad \mathbf{C}(\mathbf{k})=\left(\begin{array}{cccccc}
1 & 0 & 0 & 0 & 0 & 0 \\
0 & 1 & 0 & 0 & 0 & 0 \\
0 & 0 & 1 & 0 & 0 & 0
\end{array}\right) \\
& \mathbf{Q}(\mathbf{1})=\left(\begin{array}{cccccc}
\sigma_{x}^{2} & 0 & 0 & 0 & 0 & 0 \\
0 & \sigma_{y}^{2} & 0 & 0 & 0 & 0 \\
0 & 0 & \sigma_{\phi}^{2} & 0 & 0 & 0 \\
0 & 0 & 0 & \sigma_{v_{x}}^{2} & 0 & 0 \\
0 & 0 & 0 & 0 & \sigma_{v_{y}}^{2} & 0 \\
0 & 0 & 0 & 0 & 0 & \sigma_{\omega}^{2}
\end{array}\right) ; \quad \mathbf{Q}(\mathbf{k}>\mathbf{1})=\left(\begin{array}{cccccc}
0 & 0 & 0 & 0 & 0 & 0 \\
0 & 0 & 0 & 0 & 0 & 0 \\
0 & 0 & 0 & 0 & 0 & 0 \\
0 & 0 & 0 & \sigma_{v_{x}}^{2} & 0 & 0 \\
0 & 0 & 0 & 0 & \sigma_{v_{y}}^{2} & 0 \\
0 & 0 & 0 & 0 & 0 & \sigma_{\omega}^{2}
\end{array}\right) \\
& \mathbf{R}(\mathbf{k})=\left(\begin{array}{ccc}
\sigma_{\nu, x}^{2} & 0 & 0 \\
0 & \sigma_{\nu, y}^{2} & 0 \\
0 & 0 & \sigma_{\nu, \phi}^{2}
\end{array}\right)
\end{aligned}
$$
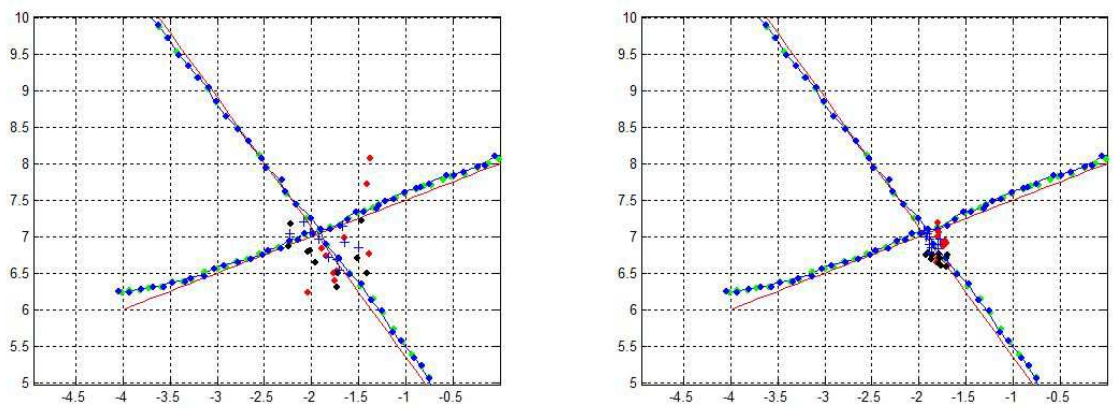

Fig. 4. Intersection - no Kalman filter

Fig. 5. Intersection - with Kalman filter

Figures 4 and 5 show the areas of intersection with the reference trajectories of robot and human (red lines) and the corresponding estimated trajectories eq.(67) from the Kalman filter. In addition we see the results for the time sequences $\mathrm{T} 1(\mathrm{k}=1 \ldots 10$,red dots $) \mathrm{T} 2(\mathrm{k}=11 \ldots 20$, black dots $)$ and $\mathrm{T} 3(\mathrm{k}=21 \ldots 30$, blue crosses). The results for the non-filtered case are quite scattered whereas the Kalman-filtered case depicts much better results for the estimated positions of the intersection. 


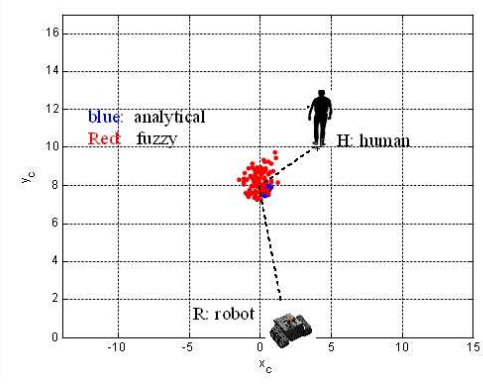

Fig. 6. Sector size: $60^{\circ}$, extracted from [18]

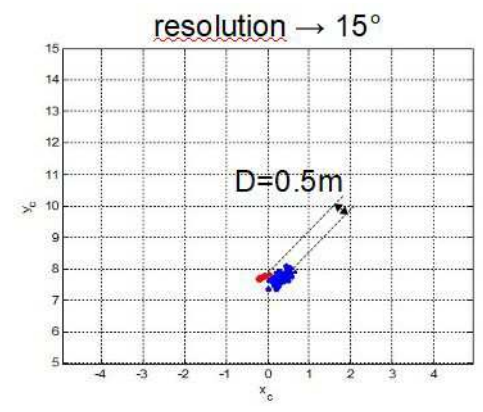

Fig. 8. Sector size: $15^{\circ}$, extracted from [18]

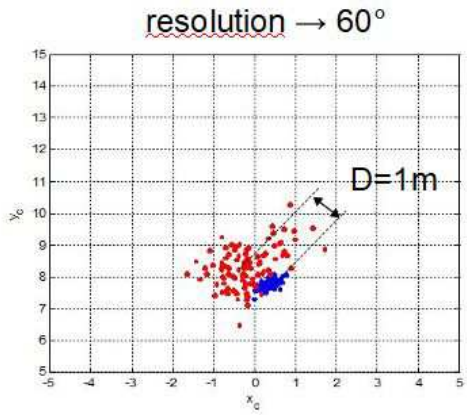

Fig. 7. Sector size: $30^{\circ}$, extracted from [18]

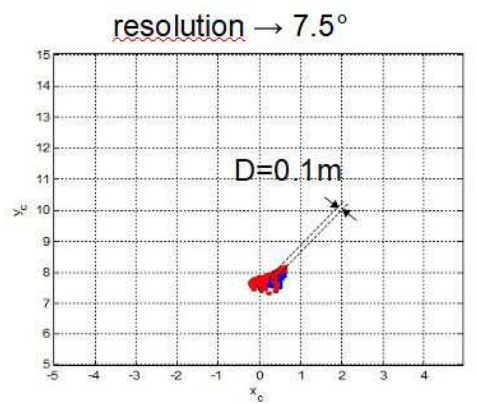

Fig. 9. Sector size: $7.5^{\circ}$, extracted from [18]

\section{Simulation results}

Gaussian input distributions The simulation results show the possibility of predicting uncertainties at possible intersections by using analytical and / or fuzzy models for a static situation in which robot and human are not moving (see fig. 1)). Position and orientation of robot and human are given by $\mathbf{x}_{R}=\left(x_{R}, y_{R}\right)=(2,0) \mathrm{m}$ and $\mathbf{x}_{H}=\left(x_{H}, y_{H}\right)=(4,10) \mathrm{m}$ and $\phi_{R}=1.78 \mathrm{rad}$, $\left(=102^{\circ}\right)$, and $\phi_{H}=3.69 \mathrm{rad},\left(=212^{\circ}\right) . \phi_{R}$ and $\phi_{H}$ are corrupted with Gaussian noise with standard deviations (std) of $\sigma_{\phi_{R}}=\sigma_{x_{1}}=0.02 \mathrm{rad},\left(=1.1^{\circ}\right)$. The fuzzy approach is compared with the analytical non-fuzzy approach by using partitions of $60^{\circ}, 30^{\circ}, 15^{\circ}, 7.5^{\circ}$ of the unit circle for the orientation angles, see table 1 and figures 6-9. Notations in table 1: $\sigma_{z_{1 c}}$ - std-computed, $\sigma_{z_{1 m}}$ - stdmeasured etc. The numbers show two qualitative results:

1. Higher resolutions lead to better results.

2. The performance with regard to a comparison between measured and computed values depends on the shape of membership functions (mf's). Lower input std's $(0.02 \mathrm{rad})$ require Gaussian mf's, higher input std's $\left(0.05 \mathrm{rad}=2.9^{\circ}\right)$ require Gaussian bell shape mf's that can be explained by different smoothing effects (see columns 4 and 5 in table 1 ). 
Results 1 and 2 can be explained by the comparison of the corresponding control surfaces and the measurements (black and red dots), see figures 10 - 14 . Figure 10 displays the control surfaces of $x_{c}$ and $y_{c}$ for the analytical case (4). The control surfaces of the fuzzy approximations (5) (see [14]) are depicted in figures 11 - 14. Starting from the resolution $60^{\circ}$ (fig. 11) we see a very high difference compared to the analytic approach (fig. 10) which decreases more and more down to resolution $7.5^{\circ}$ (fig. 14). This explains the high differences in stds and correlation coefficients in particular for sector sizes $60^{\circ}$ and $30^{\circ}$.

Table 1. Standard deviations, fuzzy and non-fuzzy results, extracted from [18]

\begin{tabular}{|c|c|c|c|c|c|c|}
\hline input std & \multicolumn{4}{|c|}{0.02 Gauss, bell shaped (GB } & Gauss & $0.05 \mathrm{~GB}$ \\
\hline sector size $/^{\circ}$ & $60^{\circ}$ & $30^{\circ}$ & $15^{\circ}$ & $7.5^{\circ}$ & $7.5^{\circ}$ & $7.5^{\circ}$ \\
\hline non-fuzz $\sigma_{z_{1 c}}$ & 0.143 & 0.140 & 0.138 & 0.125 & 0.144 & 0.366 \\
\hline fuzz $\sigma_{z_{1 c}}$ & 0.220 & 0.184 & 0.140 & 0.126 & 0.144 & 0.367 \\
\hline non-fuzz $\sigma_{z_{1 m}}$ & 0.160 & 0.144 & 0.138 & 0.126 & 0.142 & 0.368 \\
\hline fuzz $\sigma_{z_{1 m}}$ & 0.555 & 0.224 & 0.061 & 0.225 & 0.164 & 0.381 \\
\hline non-fuzz $\sigma_{z_{2 c}}$ & 0.128 & 0.132 & 0.123 & 0.114 & 0.124 & 0.303 \\
\hline fuzz $\sigma_{z_{2 c}}$ & 0.092 & 0.087 & 0.120 & 0.112 & 0.122 & 0.299 \\
\hline non-fuzz $\sigma_{z_{2} m}$ & 0.134 & 0.120 & 0.123 & 0.113 & 0.129 & 0.310 \\
\hline fuzz $\sigma_{z_{2} m}$ & 0.599 & 0.171 & 0.034 & 0.154 & 0.139 & 0.325 \\
\hline non-fuzz $\rho_{z_{12 c}}$ & 0.576 & 0.541 & 0.588 & 0.561 & 0.623 & 0.669 \\
\hline fuzz $\rho_{z_{12 c}}$ & -0.263 & 0.272 & 0.478 & 0.506 & 0.592 & 0.592 \\
\hline non-fuzz $\rho_{z_{12} m}$ & 0.572 & 0.459 & 0.586 & 0.549 & 0.660 & 0.667 \\
\hline fuzz $\rho_{z_{12} m}$ & 0.380 & 0.575 & 0.990 & 0.711 & 0.635 & 0.592 \\
\hline
\end{tabular}

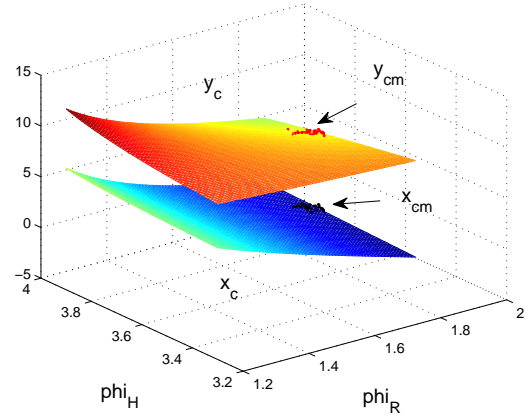

Fig. 10. Control surface non-fuzzy, extracted from [18] 


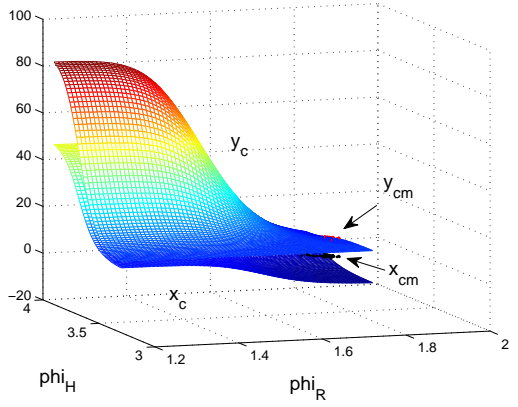

Fig. 11. Control surface fuzzy, $60^{\circ}$, extracted from [18]

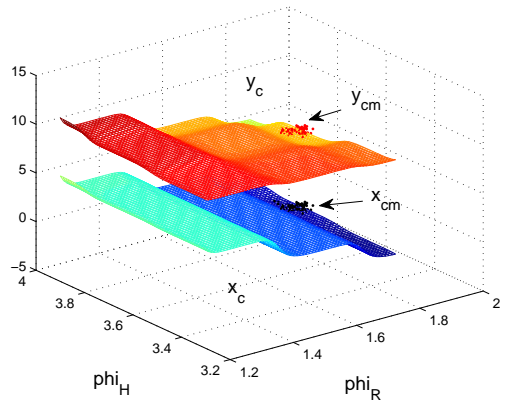

Fig. 13. Control surface fuzzy, $15^{\circ}$, extracted from $[18]$

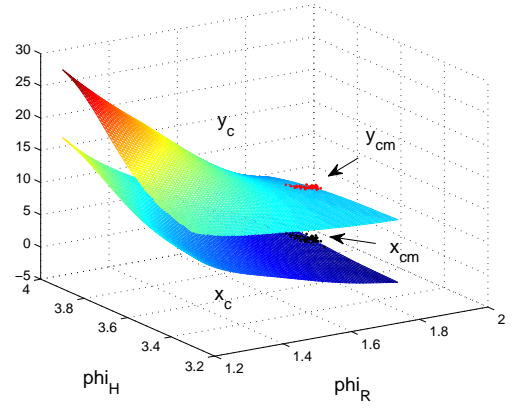

Fig. 12. Control surface fuzzy, $30^{\circ}$, extracted from $[18]$

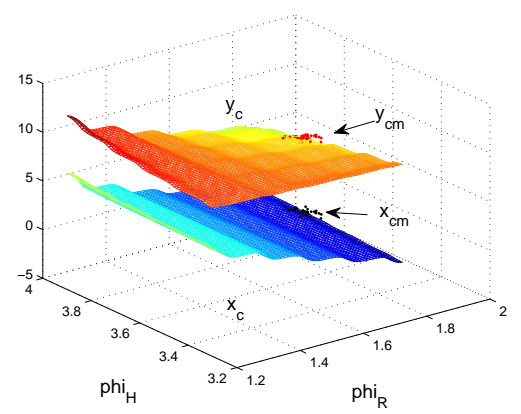

Fig. 14. Control surface fuzzy, $7.5^{\circ}$, extracted from $[18]$

\section{Energetic approach .}

In the following we concentrate on 3 examples with the following notations (see Tab.2):

stds from robot/human orientation plus position (from (45)): $\sigma_{x_{c}} ; \quad \sigma_{y_{c}}$ sum of orientation and position, computed (from (50)): $\sigma_{\text {sum }}$ sum of orientation and position, measured: $\sigma_{x r} ; \quad \sigma_{y r} ; \sigma_{s u m_{2}}$ sum of orientation and position, measured and calculated (from (40)): $\sigma_{\text {tot,end }}$ input energy (from (54)): $\sigma_{s, t o t}$

Example 1: This is a special case where all input standard deviations are equal. Positions/orientations of robot/human:

$x_{R}=2 ; \quad y_{R}=0 ; \quad x_{H}=4 ; \quad y_{H}=10 ;$

$\phi_{R}=100^{\circ}+\nu_{p h i, R} ; \quad \phi_{H}=212^{\circ}+\nu_{p h i, H} ;$

Input stds:

$\sigma_{p h i, R}=0.02 ; \sigma_{p h i, H}=0.02 ; \sigma_{x, R}=0.02 ; \sigma_{y, R}=0.02 ; \sigma_{x, H}=0.02 ; \sigma_{y, H}=0.02 ;$ In this example we see a high match between the stds $\sigma_{x_{c}}$ and $\sigma_{y_{c}}$ computed 
from (45) and the measured stds $\sigma_{x r}$ and $\sigma_{y r}$. In addition, we have a high match between $\sigma_{\text {sum }_{1}}, \sigma_{\text {sum }_{2}}, \sigma_{\text {tot }, \text { end }}$, and $\sigma_{s, t o t}$

Example 2: This example deals with slightly different input stds:

$\sigma_{p h i, R}=0.03 ; \sigma_{p h i, H}=0.05 ; \sigma_{x, R}=0.03 ; \sigma_{y, R}=0.03 ; \sigma_{x, H}=0.05 ; \sigma_{y, H}=0.05$; In this example we see also a quite high coincidence between the stds $\sigma_{x_{c}}, \sigma_{y_{c}}$ and $\sigma_{x r}, \sigma_{y r}$ mentioned above.

Example 3: This example deals with higher differences between the input stds: $\sigma_{p h i, R}=0.03 ; \sigma_{p h i, H}=0.12 ; \sigma_{x, R}=0.03 ; \sigma_{y, R}=0.03 ; \sigma_{x, H}=0.03 ; \sigma_{y, H}=0.03$; In this example we also see a fairly high match between $\sigma_{\text {sum }}, \sigma_{\text {sum }}, \sigma_{\text {tot,end }}$, and $\sigma_{s, t o t}$ but not between the stds $\sigma_{x_{c}}, \sigma_{y_{c}}$ computed from (45) and the measured stds $\sigma_{x r}$ and $\sigma_{y r}$. This result shows the advantage of the "energetic approach". The resulting standard deviation gained from the output "energy" is an average value for the intersection error that results from position/orientation errors of robot and human.

Table 2. energy approach, comparisons

\begin{tabular}{|c|c|c|c|}
\hline stds/examples & Example 1 & Example 2 & Example 3 \\
\hline \hline$\sigma_{x_{c}}$ & 0.1295 & 0.2191 & 0.2377 \\
\hline$\sigma_{y_{c}}$ & 0.1190 & 0.2783 & 0.4428 \\
\hline$\sigma_{\text {sum }_{1}}$ & 0.1759 & 0.3543 & 0.6539 \\
\hline$\sigma_{x r}$ & 0.1297 & 0.2266 & 0.2487 \\
\hline$\sigma_{y r}$ & 0.1172 & 0.2638 & 0.6032 \\
\hline$\sigma_{\text {sum }}$ & 0.1748 & 0.3478 & 0.6524 \\
\hline$\sigma_{\text {tot } \text { end }}$ & 0.1738 & 0.3433 & 0.6447 \\
\hline$\sigma_{\text {s,tot }}$ & 0.1658 & 0.3323 & 0.6084 \\
\hline
\end{tabular}

Mixed Gaussian distributions Due to larger uncertainties of the orientations of robot and human we assume the input signals to be a mixture of two Gaussian distributions with the following parameters:

$\bar{\phi}_{R 1}=1.779 \mathrm{rad},(102 \mathrm{deg}), \sigma_{\phi_{R 1}}=0.02 \mathrm{rad}$

$\bar{\phi}_{H 1}=3.698 \mathrm{rad},(212 \mathrm{deg}), \sigma_{\phi_{H 1}}=0.02 \mathrm{rad}$

$\bar{\phi}_{R 2}=1.762 \mathrm{rad},(101 \mathrm{deg}), \sigma_{\phi_{R 2}}=0.03 \mathrm{rad}$

$\bar{\phi}_{H 2}=3.716 \mathrm{rad},(213 \mathrm{deg}), \sigma_{\phi_{H 2}}=0.03 \mathrm{rad}$

$\sigma_{z_{11}}=0.1309 \mathrm{rad} ; \sigma_{z_{21}}=0.1157 \mathrm{rad}$

$\sigma_{z_{12}}=0.2274 \mathrm{rad} ; \sigma_{z_{22}}=0.1978 \mathrm{rad}$

The following computed non-fuzzy and fuzzy (superscript $F$ ) values and measured values (superscript $m$ ) according to (61) show the correctness of the previous analysis for the analytical case.

$$
\begin{array}{lll}
\bar{z}_{1}=0.487 ; & \bar{z}_{1}^{F}=0.413 ; & \bar{z}_{1}^{m}=0.485 \\
\bar{z}_{2}=7.746 ; & \bar{z}_{2}^{F}=7.737 ; & \bar{z}_{2}^{m}=7.737
\end{array}
$$




$$
\begin{aligned}
& \sigma_{z_{1}}=0.222 ; \quad \sigma_{z_{1}}{ }^{F}=0.235 ; \quad \sigma_{z_{1}}{ }^{m}=0.199 \\
& \sigma_{z_{2}}=0.184 ; \quad \sigma_{z_{2}}{ }^{F}=0.184 ; \quad \sigma_{z_{2}}{ }^{m}=0.178
\end{aligned}
$$

Figures 15 and 16 show the regarding input and output densities where Figs. 17 and 18 depict the scatter diagrams (cuts at certain density levels). Finally it turns out that the fuzzy approximation is sufficiently accurate.

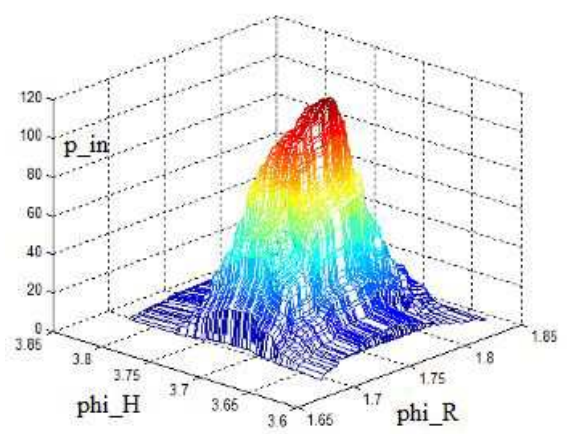

Fig. 15. Mixed Gaussian, input, extracted from [18]

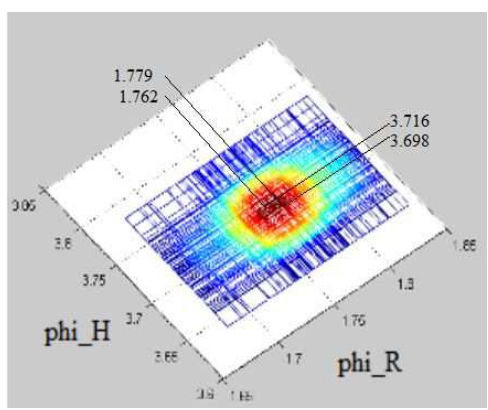

Fig. 17. Scatter diagram, mixed input, extracted from [18]

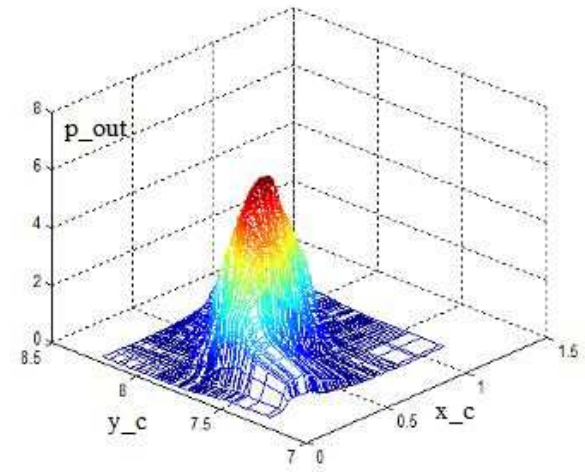

Fig. 16. Mixed Gaussian, output, extracted from [18]

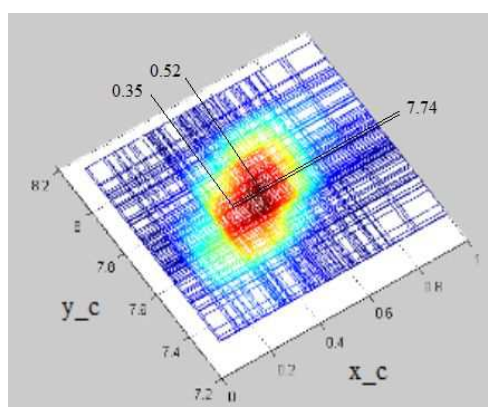

Fig. 18. Scatter diagram, mixed output, extracted from [18]

Robots and humans in motion The following simulations shows the impact of the Kalman filter on the prediction of a possible intersection between robot/human trajectories. As already mentioned in section 7 we will see the results for the measured stds $\sigma_{x_{c}}, \sigma_{y_{c}}$, and mean values $\bar{x}_{c}, \bar{y}_{c}$ of the intersection coordinates, the standard deviation $\sigma_{\text {sum }}$ that follows from the "energy" value (50). We use example 2 with the input stds of measurement noise 
$\sigma_{p h i, R}=0.03 ; \sigma_{p h i, H}=0.05 ; \sigma_{x, R}=0.03 ; \sigma_{y, R}=0.03 ; \sigma_{x, H}=0.05 ; \sigma_{y, H}=0.05 ;$ along the time sequences $\mathrm{T} 1(\mathrm{k}=1 \ldots 10), \mathrm{T} 2(\mathrm{k}=11 \ldots 20)$ and $\mathrm{T} 3(\mathrm{k}=21 \ldots 30)$. These results are then compared with the non-filtered values. The results in Table 3 show much smaller stds of the intersection coordinations for the filtered case and therefore the great benefit of Kalman filtering for positions and orientation angles. We can also see a decrease of the stds in the case of smaller distances between the intersection and the robot/human. positions. Finally fig. 19 shows the efficiency of the Kalman filter used for the orientation angle $\Phi_{H}$ of the human operator by means of which the standard deviation can be suppressed by $50 \%$ of the non-filtered case.

Table 3. example 2, stds of 3 sequences

\begin{tabular}{|c|c|c|c|c|c|c|}
\hline sequences & T1 & & T2 & & T3 & \\
\hline \hline stds & Kalman & no Kalman & Kalman & no Kalman & Kalman & no Kalman \\
\hline \hline$\sigma_{x_{c}}$ & 0.0835 & 0.1755 & 0.0803 & 0.2949 & 0.0665 & 0.2106 \\
\hline$\sigma_{y_{c}}$ & 0.2447 & 0.6840 & 0.1245 & 0.4184 & 0.1138 & 0.2208 \\
\hline$\sigma_{\text {sum }_{1}}$ & 0.2586 & 0.7062 & 0.1245 & 0.4184 & 0.1318 & 0.3051 \\
\hline $\bar{x}_{c}$ & -1.5597 & -1.4773 & -1.6186 & -1.6743 & -1.7138 & -1.6743 \\
\hline $\bar{y}_{c}$ & 6.5214 & 6.5113 & 6.3769 & 6.6380 & 6.6658 & 6.6380 \\
\hline \hline
\end{tabular}

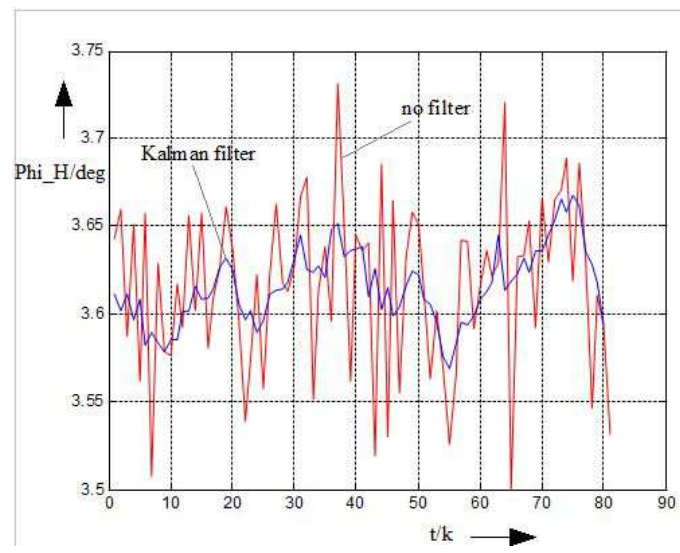

Fig. 19. Kalman filtering, orientation human

\section{Conclusions}

This research work deals with the prediction of situations and scenarios between robots and humans in shared areas for collision avoidance, task planning and control actions in the presence of uncertainties. The problem of the computation 
of intersections of human/robot trajectories is addressed, assuming that uncertainties of positions/orientations of human and robots are modeled by Gaussian noise. To do that we proposed a transformation from the human/robot positions/orientations to intersection coordinates using a geometrical model and its TS fuzzy approximation. From measured 'input' uncertainties, that are represented by standard deviations of the positions/orientations of human and robot, the 'output' standard deviations of the intersection coordinates are calculated whereas the nominal position/orientation and disturbance parameters of robot and human are supposed to be known. This analysis and its fuzzy extension applies to the static and the dynamic case provided that estimations of positions of robot and human can be derived. The method is both applied to the case of 2-inputs/2-outputs and to 6-inputs/2-outputs. In the dynamic case, Kalman filters for the estimation of robot/human positions and orientations and thus ultimately for the estimation of the intersection positions are used. For the overdetermined case, 6-inputs/2-outputs, we presented a so-called 'energetic' approach for the estimation of the intersection. The inverse task is the following: given the standard deviations for the intersection coordinates, find the corresponding input standard deviations for the orientations of robot and human. This problem is solved for the analytical and the fuzzy version of the 2-input case (orientations only). Large standard deviations of the orientation signals leads to the method of mixed Gaussian distributions. As a whole, the increase of the accuracy of human-robot pose estimations at small distances increase the system performance and human safety of human-robot collaboration which will be used in factory workshops and for robots working in rescue operations and in cooperation with human operators.

\section{Acknowledgment}

This research work has been supported by the AIR-project, Action and Intention Recognition in Human Interaction with Autonomous Systems.

\section{References}

1. Banelli, P.: Non-linear transformations of gaussians and gaussian-mixtures with implications on estimation and information theory. IEEE Trans. on Information Theory (2013)

2. Bruce, J., Wawer, J., Vaughan, R.: Human-robot rendezvous by co-operative trajectory signals. pp. 1-2 (2015)

3. Firl, J.: Probabilistic maneuver recognition in traffic scenarios. Doctoral dissertation, KIT Karlsruhe, (2014)

4. Fraichard, T., Paulin, R., Reignier, P.: Human-robot motion: Taking attention into account . Research Report, RR-8487. (2014)

5. H.Hellendoorn, R.Palm: Fuzzy system technologies at siemens r and d. Fuzzy Sets and Systens 63 (3),1994 pp. 245-259 (1994)

6. J.Chen, Wang, C., Chou, C.: Multiple target tracking in occlusion area with interacting object models in urban environments. Robotics and Autonomous Systems, Volume 103, May 2018 pp. 68-82 (2018) 
7. J.O.Smith: Signal metrics. In: Mathematics of the Fiscrete Fourier Transform (DFT) with Adio Applications. Center for Computer Research in Music and Acoustics (CCRMA) Department of Music, Stanford University, Ross Moore-Free Books, Sidney, 2000

8. J.Schaefer, K.Strimmer: A shrinkage to large scale covariance matrix estimation and implications for functional genomics. Statistical Applications in Genetics and molecular Biology, vol. 4, iss. 1, Art. 32 (2005)

9. Khatib, O.: Real-time 0bstacle avoidance for manipulators and mobile robots. IEEE Int. Conf. On Robotics and Automation,St. Loius,Missouri, 1985 p. 500505 (1985)

10. L.Foulloy, S.Galichet: Fuzzy control with fuzzy inputs. IEEE Trans. Fuzzy Systems, 11 (4) pp. 437-449 (2003)

11. O.H.Hamid, N.L.Smith: Automation, per se, is not job elimination: How artificial intelligence forwards cooperative human-machine coexistence. In: Proceedings IEEE 15th International Conference on Industrial Informatics (INDIN). pp. 899904. IEEE, Emden, Germany (2017)

12. Palm, R., Chadalavada, R., Lilienthal, A.: Fuzzy modeling and control for intention recognition in human-robot systems. In: 7. IJCCI (FCTA) 2016: Porto, Portugal (2016)

13. Palm, R., Iliev, B.: Learning of grasp behaviors for an artificial hand by time clustering and takagi-sugeno modeling. In: Proceedings FUZZ-IEEE 2006 - IEEE International Conference on Fuzzy Systems. IEEE, Vancouver, BC, Canada (July 16-21 2006)

14. Palm, R., Lilienthal, A.: Fuzzy logic and control in human-robot systems: geometrical and kinematic considerations. In: WCCI 2018: 2018 IEEE International Conference on Fuzzy Systems (FUZZ-IEEE). pp. 827-834. IEEE, IEEE (2018)

15. Pota, M., M.Esposito, Pietro, G.D.: Transformation of probability distribution into a fuzzy set interpretable with likelihood view. In: IEEE 11th International Conf. on Hybrid Intelligent Systems (HIS 2011). pp. 91-96. IEEE, Malacca Malaysia (2011)

16. R.Palm, Driankov, D.: Tuning of scaling factors in fuzzy controllers using correlation functions. In: Proceedings FUZZ-IEEE'93. IEEE, IEEE, San Francisco, california (1993)

17. R.Palm, Driankov, D.: Fuzzy inputs. Fuzzy Sets and Systems - Special issue on modern fuzzy control pp. 315-335 (1994)

18. R.Palm, Lilienthal, A.: Uncertainty and fuzzy modeling in human-robot navigation. In: Proc. of the 11th Intern. Joint Conf. on Comp. Int. (IJCCI 2019), pp. 296-305. IJCCI, SCITEPRESS, Wien

19. Tahboub, K.A.: Intelligent human-machine interaction based on dynamic bayesian networks probabilistic intention recognition. Journal of Intelligent and Robotic Systems. Volume 45, Issue 1, 31-52 (2006)

20. W.Luo, J.Xing, Milan, A., Zhang, X., Liu, W., Zhao, X., Kim, T.: Multiple object tracking: A literature review. Computer Vision and Pattern Recognition, arXiv 1409,7618 p. 118 (2014)

21. Yager, R., Filev, D.B.: Reasoning with probabilistic inputs. In: Proceedings of the Joint Conference of NAFIPS, IFIS and NASA. pp. 352-356. NAFIPS, San Antonio (1994) 